\title{
Diversity of human papillomavirus in the anal canal of HIV-positive and HIV-negative men
}

\author{
Luisa Galatia, Rosario N. Brancaccio a , Purnima Gupta ${ }^{a, 1}$, Eugenie Lohmann ${ }^{a}$, \\ Alexis Robitaille $^{\mathrm{a}, 2}$, Racheal S. Dube Mandishora ${ }^{\mathrm{a}, \mathrm{b}}$, Cyrille Cuenin ${ }^{\mathrm{a}}$, Raffaele Filotico ${ }^{\mathrm{c}}$, \\ Jean-Damien Combes a , Anna R. Giuliano ${ }^{\mathrm{d}}$, Maria Gabriella Donà ${ }^{\mathrm{e}}$, Massimo Tommasino ${ }^{\mathrm{a}, *}$, \\ Tarik Gheit ${ }^{\mathrm{a}, *}$
}

\footnotetext{
a International Agency for Research on Cancer, 150 cours Albert Thomas, 69372 Lyon Cedex 08, France

${ }^{\mathrm{b}}$ Department of Medical Microbiology, University of Zimbabwe College of Health Sciences, Harare, Zimbabwe

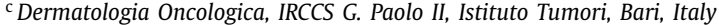

${ }^{\mathrm{d} C e n t e r}$ for Immunization and Infection Research in Cancer, Moffitt Cancer Center, Tampa, FL, USA

e STI/HIV Unit, San Gallicano Dermatological Institute IRCCS, Rome, Italy
}

\section{A R T I C L E I N F O}

\section{Article history:}

Accepted 24 November 2020

Available online 28 November 2020

\section{Keywords: \\ HPV \\ MSM \\ MSW \\ Anal canal \\ NGS \\ HIV}

\begin{abstract}
S U M M A R Y
Background: To characterize the HPV diversity in the anal mucosa of men with different sexual behavior and HIV status by next-generation sequencing (NGS).

Methods: Anal swabs from HIV-positive ( $n=94$; mean age, 38 years) and HIV-negative $(n=100$; mean age, 37.5 years) men who have sex with men (MSM) and HIV-negative men (predominantly men who have sex with women, MSW) ( $n=99$; mean age, 38.2 years) were analyzed by broad-spectrum PCR protocols combined with NGS.

Findings: Alpha HPV types $(n=74)$ were detected mainly in the MSM groups (HPV6, 11 , and 43 were the most abundant types) compared with MSW $(n=16)$ (HPV11, 32, and 87 were among the most abundant). In contrast, beta HPVs were more abundantly detected among MSW $(n=45)$ than in the HIVpositive $(n=16)$ and HIV-negative $(n=26)$ MSM groups. Gamma HPVs were detected almost equally in HIV-positive MSM $(n=62)$, HIV-negative MSM $(n=58)$, and MSW $(n=57)$. In addition, 31 putative novel PV types were identified.

Conclusions: Our data show that beta and gamma HPV types are present in the anal mucosa, thus reinforcing the existing evidence that they can be detected at anatomical sites other than skin. Alpha and beta HPV distribution among these three groups appears to vary according to sexual behavior.
\end{abstract}

(C) 2020 Published by Elsevier Ltd on behalf of The British Infection Association.

\section{Introduction}

Human papillomaviruses (HPVs) are classified into five genera: alpha ( $n=65$ types), beta ( $n=54$ types), gamma ( $n=99$ types), mu ( $n=3$ types), and nu ( $n=1$ type) (www.hpvcenter.se, accessed on 10-08-2020). ${ }^{1}$ Twelve mucosal alpha HPV types $(16,18,31,33,35$, $39,45,51,52,56,58$, and 59) are classified as high-risk (HR-HPV) and have been associated with anogenital cancers and a subset of head and neck cancers by the International Agency for Research

\footnotetext{
* Corresponding authors.

E-mail addresses: tommasinom@iarc.fr, icb@iarc.fr (M. Tommasino), gheitt@iarc.fr (T. Gheit).

1 Present address: De Duve Institute, GECE, Avenue Hippocrate 74/B1.74.04, 1200 Woluwe-Saint-Lambert, Brussels, Belgium.

2 Present address: Heinrich Pette Institute, Leibniz Institute for Experimental Virology, Hamburg, Germany.
}

on Cancer (IARC) Monographs evaluations. ${ }^{2}$ Beta HPVs appear to cooperate with ultraviolet radiation in the development of cutaneous squamous cell carcinoma. ${ }^{2}$ Beta HPVs are subdivided into five species (beta- 1 to beta-5). The beta- 1 and beta- 2 species include the majority of beta types that are abundantly present in the skin of healthy individuals. ${ }^{3}$ In contrast, beta-3-5 HPV types are less frequently detected in the skin. Epidemiological studies indicate that beta HPV types are also present at other anatomical sites, such as oral and nasal mucosa, ${ }^{4-6}$ suggesting a dual tissue tropism of certain HPV types. Some studies found beta- 3 HPV s to be more prevalent than beta- 1 and beta- 2 HPVs in mucosal epithelia, including the anal canal. ${ }^{3}$ Interestingly, in vivo and in vitro experimental models have shown that beta-3 HPV49 shares biological properties with mucosal HR-HPV types. ${ }^{7}$

The largest genus (i.e., gamma) includes the majority of discovered HPV types, which are subdivided into 27 species. How- 
ever, no studies to date have evaluated their possible link with human carcinogenesis, with the exception of a functional study that highlighted the transforming properties of gamma-24 HPV197 in in vitro experimental models. ${ }^{8}$

Several studies have highlighted the utility of next-generation sequencing (NGS) for HPV characterization.9,10 However, these studies focused mainly on NGS-based assays for the detection of HR-HPV types in the anal mucosa. ${ }^{11-13}$

To better characterize HPV diversity in anal mucosal epithelia, we used broad-spectrum PCR protocols combined with NGS assay, analyzing anal swabs collected from HIV-positive and HIV-negative men who have sex with men (MSM) as well as a subgroup of HIV-negative men, predominantly men who have sex with women (MSW), from the HPV Infection in Men (HIM) Study.

\section{Methods}

\section{Study population and biological specimens}

In the present study, we analyzed a total of 293 anal canal specimens obtained from men enrolled in previously published studies. ${ }^{3,14,15}$ Briefly, HIV-positive $(n=94)$ and HIV-negative $(n=100)$ MSM were randomly selected and matched by age (mean age, 38 and 37.5 years, respectively) from among the attendees of the STI/HIV Unit of the San Gallicano Dermatological Institute (Rome, Italy). In addition, 99 anal swabs were obtained from men in the HIM Study, a large, multinational (Brazil, Mexico, and the USA) prospective cohort study investigating the natural history of HPV infection in HIV-negative men. ${ }^{15}$ The HIM specimens were selected according to age and sample size to match with other groups included in the study. All enrolled participants (mean age, 38.2 years) were HIV-negative, and most (91\%) were MSW; only $5 \%$ were men who have sex with both men and women, $2 \%$ were MSM, and $2 \%$ had never had sex or the information was not available.

\section{NGS broad-spectrum analysis and bioinformatic analysis}

Extracted DNA from anal samples was amplified by PCR using two sets of primers: (i) FAP59/64 ${ }^{16}$ and (ii) FAPM1 primer sets, as previously described. ${ }^{17}$ PCR amplicons of approximately $480 \mathrm{bp}$ were purified using the QIAquick gel extraction purification kit according to the manufacturer's instructions (QIAGEN, Hilden, Germany) and divided into six different pools, according to HIV status, enrolled groups, and PCR protocol applied, as indicated in Table 1. An additional purification step was performed in each pool, and
NGS libraries were prepared using the Nextera ${ }^{\mathrm{TM}}$ DNA Flex Library preparation kit (Illumina, San Diego, CA, USA) as previously described. ${ }^{17,18}$

Bioinformatic analysis was performed using the bioinformatic pipeline PVAmpliconFinder. ${ }^{19}$ All the results in this study were based on the identification of the sequences following the homology-based classification using the evolutionary placement algorithm (EPA) in RAxML (Randomized Axelerated Maximum Likelihood). Only the longest sequence was considered for RAxML-EPA classification when several singlets or contigs were clustered together by PVAmpliconFinder.

\section{Results}

\section{HPV DNA PCR amplification and NGS analyzes}

To determine whether HPV infection in the anal canal may differ in different groups of men, we evaluated the distribution in (i) HIV-negative MSM $(n=100)$, (ii) HIV-positive MSM $(n=94)$, and (iii) predominantly MSW $(n=99)$. The total number of samples yielding an amplicon of the expected size by both FAP and FAPM1 primers are shown in Table 1. Amplicons for each group were then pooled and analyzed by NGS.

A higher number of alpha HPV reads was found among MSM compared with MSW, independently of HIV status (Table 2). In both MSM groups, the total number of reads corresponded to 37 alpha HPV types (Table 2), with some HPV types detected in both groups with the two PCR protocols. Among MSW, the total number of alpha HPV reads corresponded to 16 HPV types (Table 2 and Fig. 1). The representative alpha species found in both MSM groups were alpha- $1,-3,-5,-6,-7,-8,-9,-10,-11,-13$, and -14 (Supplementary file S1). Comparing the two MSM groups, reads of alpha types HPV6, 16, 30, 34, 39, 43, 58, 59, 70, 81, 85, 86, 87, 90 , and 114 were detected more in HIV-positive MSM pools, and reads of HPV $11,18,26,31,32,40,42,45,51,52,53,54,62$, $68,71,73,84,89$, and 91 were represented more in HIV-negative MSM pools (Supplementary file S1). A small number of HPV69, 83, and 102 reads were detected in the HIV-positive MSM group only, and HPV61, 67, and 82 reads in the HIV-negative MSM group only (Fig. 1 and Supplementary file S1). Among MSW, the alpha HPV reads corresponded to species alpha- $1,-2,-3,-6,-7,-9,-10$, -13 , and -14 , with the more represented HPV types being HPV11, 16, 32, 42, 68, and 87 (Supplementary file S1). Overall, 14 alpha HPVs were present in all three groups (HIV-positive MSM, HIVnegative MSM, and MSW), namely HPV11, 16, 18, 30, 32, 42, 54,
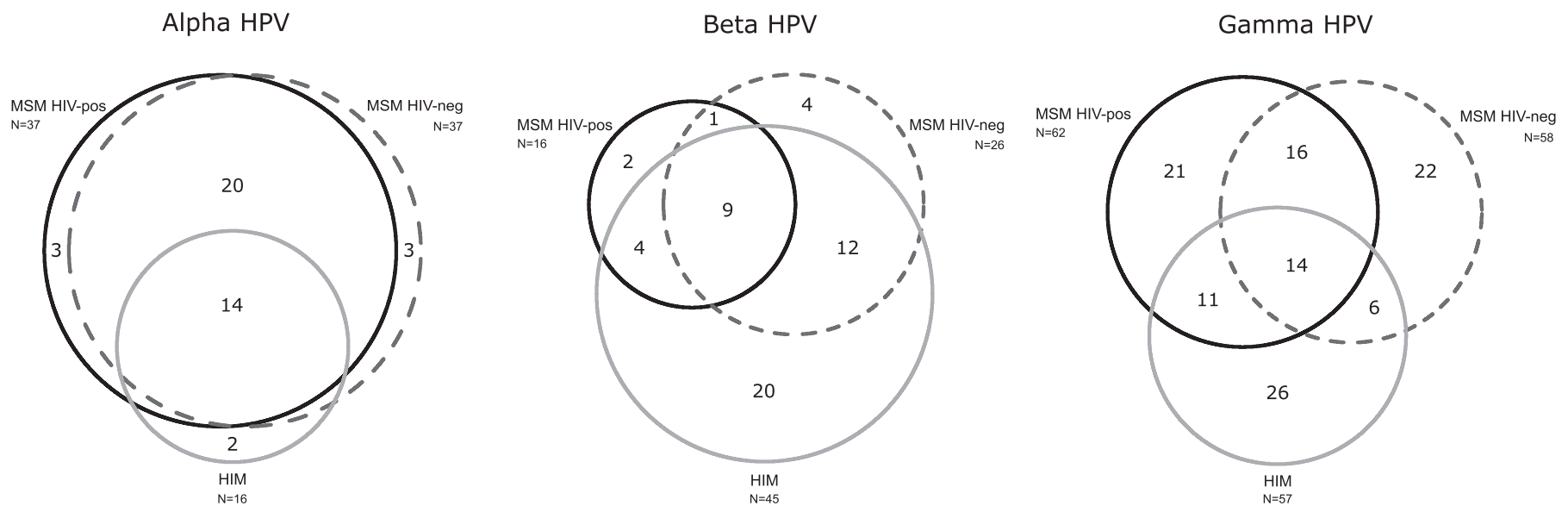

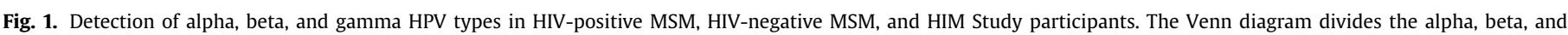
gamma HPV types into MSM HIV-positive, MSM HIV-negative, and HIM groups. 
Table 1

Total number of samples from which amplicons of the expected size were obtained, grouped according to the PCR protocols and the participant groups.

\begin{tabular}{llll}
\hline NGS pool & PCR protocol & Specimens & $N(\%)$ \\
\hline 1 & FAP59/64 & HIV-positive MSM & $75(79.8)$ \\
2 & FAP59/64 & HIV-negative MSM & $52(52.0)$ \\
3 & FAP59/64 & HIM & $50(50.5)$ \\
4 & FAPM1 & HIV-positive MSM & $75(79.8)$ \\
5 & FAPM1 & HIV-negative MSM & $67(67.0)$ \\
6 & FAPM1 & HIM & $41(41.4)$ \\
\hline
\end{tabular}

Positive PCR products.

$59,62,68,84,87,90$, and 114 , and two were present only among MSW (HPV3 and 74) (Fig. 1 and Supplementary file S1).

In contrast to alpha HPV types, beta HPVs $(n=45)$ were more common among MSW with both PCR protocols than in the MSM groups (Fig. 1). In addition, HIV positivity correlated with a lower number of beta HPV types $(n=16)$ compared with HIV-negative MSM $(n=26)$ (Fig. 1, Supplementary file S1). All five beta species were represented in the three groups (Supplementary file S1).

Fig. 1 shows that 20 beta HPV types were present only among MSW, which corresponded to HPV5, 8, 17, 21, 25, 47, 76, 80, $98,100,111,113,118,145,159,150,217$, and three unreferenced beta PVs represented by HPV-mm292c14, HPV-mw15c111, and HPVmTVMBSGc2024 (Supplementary file S1). In addition, 7 beta HPV types were present only among MSM (HPV15, 99, $105,151,152$, and two unreferenced HPVs represented by HPVmTVMBSFc09 and HPV-mm292c100). Nine HPV types were present in all three groups of men (HPV20, 23, 37, 49, 96, 107, 115, 120, and one unreferenced HPV, that is HPV-mHIVGc36) (Fig. 1, Supplementary file S1).

Gamma HPVs were detected approximately equally in all three groups (Table 2) and corresponded to 62, 58, and 57 gamma HPV types in HIV-positive MSM, HIV-negative MSM, and MSW, respectively (Table 2 and Fig. 1 ). Reads from gamma-10, $-8,-7$, and -6 species were the most abundant in the HIV-positive MSM group. Gamma-10, $-6,-11$, and -27 species were abundantly detected in the HIV-negative MSM group, and gamma-9, $-10,-20$, and -26 species showed the highest number of reads among MSW.

Fourteen gamma HPV types (HPV50, 88, 103, 108, 130, 135, $142,146,154,163,180$, HPV-mHIVGc70, HPV-mSK250, and HPV-mw20c01b) were present in all three groups. Twenty-one gamma types were present only in the HIV-positive MSM group (HPV109, 119, 147, 153, 165, 211, 216, HPV-mKN3, HPV-mSE383, HPV-mSK006, HPV-mSK020, HPV-mSK085, HPV-mSK102, HPVmSK104, HPV-mSK128, HPV-mSK131, HPV-mSK193, HPV-mSK243, HPV-mw20c04, HPV-mw27c04c, and HPV-mw34c14a) (Fig. 1 and Supplementary file S1). Twenty-two gamma types were present only among HIV-negative MSM (HPV148, 155, 157, 161, 169, 197, HPV-m16031680A, HPV-mEV03c45, HPV-mKC5, HPV-mSE355, HPV-mSK009, HPV-mSK029, HPV-mSK038, HPV-mSK064, HPVmSK083, HPV-mSK197, HPV-mSK199, HPV-mSK242, HPV-mSK245, HPV-mTVMBSGc2450, HPV-mw18c11d, and HPV-mw20c09). Finally, 26 gamma types were present only among MSW (HPV4, 65, 112, 128, 131, 172, 179, 203, HPV-mEV03c212, HPV-mEV03c40, HPV-mSK052, HPV-mSK056, HPV-mSK061, HPV-mSK072, HPVmSK075, HPV-mSK094, HPV-mSK096, HPV-mSK101, HPV-mSK126, HPV-mSK190, HPV-mSK244, HPV-mTVMBSHc33, HPV-mw11C24, HPV-mw11c13, HPV-mw18c39, and HPV-mw34c34a) (Fig. 1 and Supplementary file $\mathrm{S} 1$ ).

A total of $5892(0.2 \%)$ reads were classified as putative novel PVs $(n=31)$, because their sequences showed less than $90 \%$ similarity to the L1 open reading frame (ORF) of any known PVs (Supplementary file S2). According to RAxML-EPA classification, the majority of putative novel PVs were closely related to gamma $(n=24)$, alpha $(n=3)$, and beta $(n=2)$ HPV genera, whereas a subset of reads $(n=360)$ were representative of two putative PVs related to one unclassified PV. The latter were found in HIV-positive MSM (Supplementary file S2).

\section{Discussion}

In this study, we analyzed the presence of a broad spectrum of HPV types in anal swabs from HIV-negative and HIV-positive MSM and HIV-negative MSW. In agreement with previous studies, ${ }^{20-23}$ our results revealed the presence of a large number of alpha, beta, and gamma types in all groups, further supporting the concept that beta and gamma HPV types have the ability to infect both cutaneous and mucosal epithelia. Hampras et al. reported that HPV types of the beta- 3 species were more likely to infect the anal mucosa compared with other anatomical sites, such as oral mucosa or skin epithelia. ${ }^{3}$ Moreover, Bolatti et al. reported that gamma PVs may also display dual tissue tropism, being highly prevalent in anal mucosa. ${ }^{21}$ Our data show that the abundance of the specific HPV genera appears to differ in the three groups of men. Indeed, alpha HPV types were more abundantly detected in MSM than in MSW. Regarding the presence of specific alpha HPV types, HPV6, 11 , and 43 were the most abundant types in both MSM groups. Reads from the low-risk HPV11 type were also among the most abundant among MSW, together with HPV32 and 87. The presence of a higher number of alpha HPV types among MSM compared with MSW may be explained by the different sexual behaviors and is in agreement with previous studies. ${ }^{23,24}$ Infections with mucosal high-risk alpha HPVs account for $90 \%$ of anal cancer cases worldwide. An increased risk of anal cancer has been clearly shown in HIV-positive individuals and MSM. ${ }^{25}$ In contrast to alpha HPV types, beta HPV types were less abundant among MSM compared with MSW, whereas gamma HPV types were detected at similar abundances in the MSM and MSW. The presence of HPVs in the anal canal of MSW may reflect other ways of transmission compared with those for MSM. ${ }^{24,26}$ Previous studies have shown that the prevalence of anal beta HPVs increases with the number of lifetime sexual partners. ${ }^{27}$ In addition, a high prevalence of beta HPVs in a cohort of MSW was previously reported. ${ }^{24}$ This apparent difference in distribution of alpha and beta HPVs in MSM and MSW may be explained by the fact that alpha HPVs may interfere in some way with beta HPVs in the anal canal. Although unlikely, the different distribution of the two HPV genera in MSM and MSW might be linked to the PCR protocol used in the study. It is possible that a smaller number of beta HPV types could be amplified by the FAP PCR protocols in specimens from MSM compared with those from MSW, due to the abundance of alpha HPV types. However, this hypothesis is not supported by the fact that gamma HPV types were detected at similar abundances in the three groups using primers that were originally designed by considering most HPV types.

Among gamma types, reads from gamma-10 HPV121 were among the most abundant referenced HPV types among HIVpositive and HIV-negative MSM. In addition, gamma-6 HPV103 


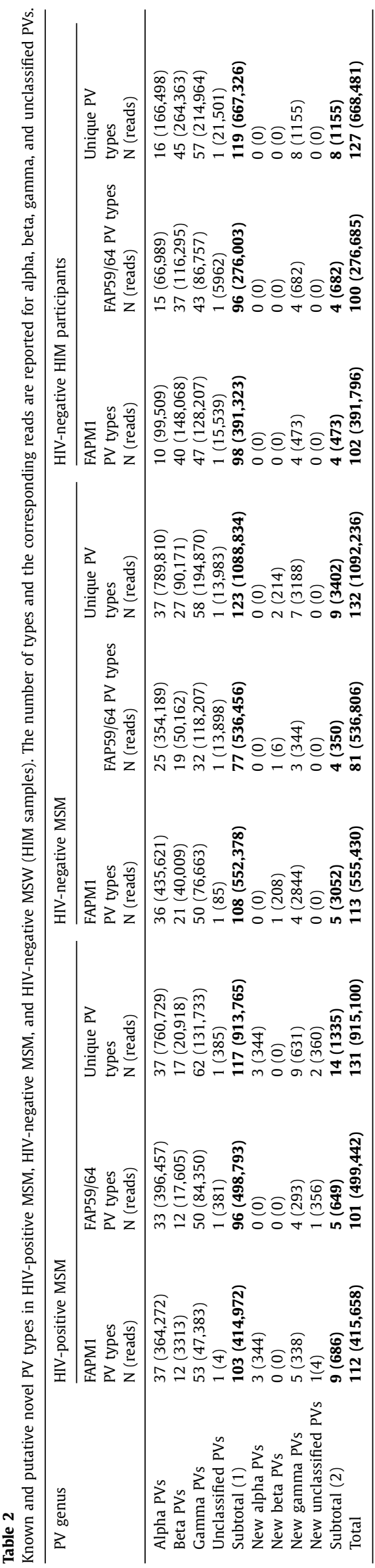

showed the highest number of reads in HIV-negative MSM. Gamma-9 HPV215 was the most abundant among MSW.

HPVs belonging to the gamma- 6 species have previously been suggested to display a preferential tropism for mucosal tissue. ${ }^{21,28}$ In support of this, gamma-6 types were not detected in our previous NGS study carried out on skin specimens, ${ }^{18}$ or in other similar studies. $^{29}$

Finally, we also identified 31 putative new HPV types. Most of them $(n=24)$ were assigned to the gamma genus, suggesting that even more gamma PV types can be detected in the anal mucosa.

A large number of gamma HPVs were discovered during the past decade, and all are poorly characterized. Experimental and epidemiological studies regarding the presence of these gamma PV types in mucosal epithelia are required to evaluate their role in human pathological conditions. Most of the putative new PVs were identified in the HIV-positive MSM; this may be a consequence of the possible impact of HIV infection on the immune system, although the majority of the HIV-positive MSM included in this study were using combination antiretroviral therapy. Still, it is known that HIV-positive individuals are highly susceptible to HPV infections. $^{30}$ Three putative new alpha HPVs were found in this group, both related to low-risk HPV types, i.e. HPV6 and 89. However, the fact that our study was based on a pool-based strategy is a limitation and does not allow the determination of any specific prevalence of HPV types across the three analyzed groups. Although stringent protocols were adopted for the collection of anal specimens, due to the high sensitivity of NGS, it is not possible to exclude that some HPV sequences derived from the perianal region. In addition, in our study we compared populations from different geographical regions. Future studies should consider MSW and MSM groups from the same geographical areas.

According to the applied bioinformatics workflow, we identified animal viruses in the anal canal. However, the classification of these PVs as animal types could be due to the limited information on the HPV L1 sequence, and the consideration of additional parts of the genome could result in a different classification as closely related HPVs. ${ }^{19}$ In conclusion, using a well-validated NGS strategy, this study expanded our knowledge on HPV diversity in the anal mucosa. It showed that, besides infections with alpha HPVs, many beta and gamma HPV types from different species colonize the anal mucosal epithelium. In agreement with previous studies, the beta- 3 and gamma- 6 species appear to be abundantly present at this anatomical site., ${ }^{3,21}$ In addition, this strategy enabled the detection of putative novel PVs. Overall, this study confirmed the high diversity of HPVs in anal samples of male participants, irrespective of their sexual behaviors. A different distribution of alpha and beta genera in MSM compared with mostly MSW was observed. Finally, these results reinforced the existing evidence of a dual tropism of many beta or gamma so-called cutaneous HPV types in the anal canal.

\section{Declaration of Competing Interest}

Dr Giuliano has received compensation for her role as a consultant and member of Merck \& Co., Inc. Advisory Boards. Her institution has received funding for research. No conflicts of interest are declared for any other authors.

\section{Acknowledgments}

We are grateful to all members of our laboratories for their cooperation and to Nicole Suty for her help with manuscript preparation ad Dr Karen Muller for editing. We are also grateful to Alessandra Latini, Massimo Giuliani, Amalia Giglio, Maria Benevolo, and Francesca Rollo, who are participating in the Italian study on MSM groups. 


\section{Funding}

No external funding was received for this study.

\section{Disclaimer}

Where authors are identified as personnel of the International Agency for Research on Cancer/World Health Organization, the authors alone are responsible for the views expressed in this article and they do not necessarily represent the decisions, policy, or views of the International Agency for Research on Cancer/World Health Organization.

\section{Ethics approval}

The study was cleared by the local ethics committee (CE/564/11 and CE/436/14) for the San Gallicano Dermatological Institute IRCCS, and by the committees of the University of South Florida, USA, the Centro de Referência e Tratamento de Doenças Sexualmente Transmissiveis e AIDS, Brazil, and the National Institute of Public Health of Mexico, and was performed in accordance with the principles of the Declaration of Helsinki.

\section{Supplementary materials}

Supplementary material associated with this article can be found, in the online version, at doi:10.1016/j.jinf.2020.11.030.

\section{References}

1. Bernard HU, Burk RD, Chen Z, van Doorslaer K, zur Hausen H, de Villiers EM. Classification of papillomaviruses (PVs) based on 189 PV types and proposal of taxonomic amendments. Virology 2010;401(1):70-9. doi:10.1016/j.virol.2010.02. 002 .

2. Biological agents Volume 100 B a review of human carcinogens. IARC monographs on the evaluation of carcinogenic risks to humans/World Health Organization. Int Agency Res Cancer 2012:100(Pt B):1-441.

3. Hampras SS, Rollison DE, Giuliano AR, McKay-Chopin S, Minoni L, Sereday K, et al. Prevalence and concordance of cutaneous beta human papillomavirus infection at mucosal and cutaneous sites. J Infect Dis 2017;216(1):92-6. doi:10. 1093/infdis/jix245

4. Wong MCS, Vlantis AC, Liang M, Wong PY, Ho WCS, Boon SS, et al. Prevalence and epidemiologic profile of oral infection with alpha, beta, and gamma papillomaviruses in an Asian Chinese population. J Infect Dis 2018;218(3):388-97. doi:10.1093/infdis/jiy160.

5. Forslund O, Johansson H, Madsen KG, Kofoed $\mathrm{K}$. The nasal mucosa contains a large spectrum of human papillomavirus types from the Betapapillomavirus and Gammapapillomavirus genera. J Infect Dis 2013;208(8):1335-41. doi:10.1093/ infdis/jit326.

6. Winer RL, Gheit T, Feng Q Stern JE, Lin J, Cherne S, Tommasino M. Prevalence and correlates of $\beta$ - and $\gamma$-human papillomavirus detection in oral samples from mid-adult women. J Infect Dis 2019;219(7):1067-75. doi:10.1093/infdis/ jiy632.

7. Cornet I, Bouvard V, Campo MS, Thomas M, Banks L, Gissmann L, et al Comparative analysis of transforming properties of E6 and E7 from different beta human papillomavirus types. J Virol 2012;86(4):2366-70. doi:10.1128/JVI. 06579-11.

8. Grace M, Munger K. Proteomic analysis of the gamma human papillomavirus type 197 E6 and E7 associated cellular proteins. Virology 2017;500:71-81. doi:10.1016/j.virol.2016.10.010.

9. Yin L, Yao J, Chang K, Gardner BP, Yu F, Giuliano AR, et al. HPV population profiling in healthy men by next-generation deep sequencing coupled with HPVQUEST. Viruses 2016;8(2). doi:10.3390/v8020028.

10. Meiring TL, Salimo AT, Coetzee B, Maree HJ, Moodley J, Hitzeroth II, et al. Nextgeneration sequencing of cervical DNA detects human papillomavirus types not detected by commercial kits. Virol J 2012;9:164. doi:10.1186/1743-422x-9-164.

11. Nowak RG, Ambulos NP, Schumaker LM, Mathias TJ, White RA, Troyer J, et al. Genotyping of high-risk anal human papillomavirus (HPV): ion torrentnext generation sequencing vs. linear array. Virol J 2017;14(1):112. doi:10.1186/ s12985-017-0771-z.

12. Piyathilake CJ, Badiga S, Kumar R, Crowley MR, Burkholder GA, Raper JL. A rigorous exploration of anal HPV genotypes using a next-generation sequencing (NGS) approach in HIV-infected men who have sex with men at risk for developing anal cancer. Cancer Med 2020;9(2):807-15. doi:10.1002/cam4.2720.

13. Tsikis S, Hoefer L, Bethimoutis G, Nicolaidou E, Paparizos V, Antoniou C, et al. Risk factors, prevalence, and site concordance of human papillomavirus in highrisk Greek men. Eur J Cancer Prev Off J Eur Cancer Prev Organ 2018;27(5):514-20. doi:10.1097/cej.0000000000000366.
14. Dona MG, Gheit T, Latini A, Benevolo M, Torres M, Smelov V, et al. Alpha, beta and gamma human papillomaviruses in the anal canal of HIV-infected and uninfected men who have sex with men. J Infect 2015;71(1):74-84. doi:10.1016/j. jinf.2015.02.001.

15. Giuliano AR, Lazcano-Ponce E, Villa LL, Flores R, Salmeron J, Lee JH, et al. The human papillomavirus infection in men study: human papillomavirus prevalence and type distribution among men residing in Brazil, Mexico, and the United States. Cancer Epidemiol Biomark Prev 2008;17(8):2036-43. doi:10.1158/ 1055-9965.Epi-08-0151.

16. Forslund O, Antonsson A, Nordin P, Stenquist B, Hansson BG. A broad range of human papillomavirus types detected with a general PCR method suitable for analysis of cutaneous tumours and normal skin. J Gen Virol 1999;80(Pt 9):243743. doi:10.1099/0022-1317-80-9-2437.

17. Brancaccio RN, Robitaille A, Dutta S, Cuenin C, Santare D, Skenders G, et al Generation of a novel next-generation sequencing-based method for the isolation of new human papillomavirus types. Virology 2018;520:1-10. doi:10.1016/j. virol.2018.04.017.

18. Galati L, Brancaccio RN, Robitaille A, Cuenin C, Luzi F, Fiorucci G, et al. Detection of human papillomaviruses in paired healthy skin and actinic keratosis by next generation sequencing. Papillomavirus Res Amst Neth 2020;9:100196. doi:10.1016/j.pvr.2020.100196.

19. Robitaille A, Brancaccio RN, Dutta S, Rollison DE, Leja M, Fischer N, et al. PVAmpliconfinder: a workflow for the identification of human papillomaviruses from high-throughput amplicon sequencing. BMC Bioinform 2020;21(1):233. doi:10. 1186/s12859-020-03573-8.

20. Mlakar B, Kocjan BJ, Hosnjak L, Fujs Komlos K, Milosevic M, Poljak M. Betapapillomaviruses in the anal canal of HIV positive and HIV negative men who have sex with men. J Clin Virol 2014:61(2):237-41. doi:10.1016/j.jcv.2014.07.009.

21. Bolatti EM, Hošnjak L, Chouhy D, Casal PE, Re-Louhau MF, Bottai H, et al. Assessing gammapapillomavirus infections of mucosal epithelia with two broad-spectrum PCR protocols. BMC Infect Dis 2020;20(1):274. doi:10.1186/ s12879-020-4893-3.

22. Li X, Li M, Yang Y, Zhong X, Feng B, Xin H, et al. Anal HPV/HIV co-infection among men who have sex with men: a cross-sectional survey from three cities in China. Sci Rep 2016:6:21368. doi:10.1038/srep21368.

23. Donà MG, Vescio MF, Latini A, Giglio A, Moretto D, Frasca M, et al. Anal human papillomavirus in HIV-uninfected men who have sex with men: incidence and clearance rates, duration of infection, and risk factors. Clin Microbiol Infect Off Publ Eur Soc Clin Microbiol Infect Dis 2016;22(12):1004 e1-.e7. doi:10.1016/j.cmi. 2016.08.011

24. Sichero L, Nyitray AG, Nunes EM, Nepal B, Ferreira S, Sobrinho JS, et al. Diversity of human papillomavirus in the anal canal of men: the HIM study. Clin Microbio Infect Off Publ Eur Soc Clin Microbiol Infect Dis 2015;21(5):502-9. doi:10.1016/j. cmi.2014.12.023.

25. Clifford GM, Georges D, Shiels MS, Engels EA, Albuquerque A, Poynten IM, et al A meta-analysis of anal cancer incidence by risk group: toward a unified anal cancer risk scale. Int J Cancer 2020. doi:10.1002/ijc.33185.

26. Pamnani SJ, Nyitray AG, Abrahamsen M, Rollison DE, Villa LL, Lazcano-Ponce E et al. Sequential acquisition of anal human papillomavirus (HPV) infection following genital infection among men who have sex with women: the HPV infection in men (HIM) study. J Infect Dis 2016;214(8):1180-7. doi:10.1093/infdis/ jiw334.

27. Smelov V, Muwonge R, Sokolova O, McKay-Chopin S, Eklund C, Komyakov B, et al. Beta and gamma human papillomaviruses in anal and genital sites among men: prevalence and determinants. Sci Rep 2018;8(1):8241. doi:10.1038/ s41598-018-26589-w.

28. Chen Z, Schiffman M, Herrero R, Desalle R, Burk RD. Human papillomavirus (HPV) types 101 and 103 isolated from cervicovaginal cells lack an E6 open reading frame (ORF) and are related to gamma-papillomaviruses. Virology 2007;360(2):447-53. doi:10.1016/j.virol.2006.10.022.

29. Bolatti EM, Hosnjak L, Chouhy D, Re-Louhau MF, Casal PE, Bottai H, et al High prevalence of gammapapillomaviruses (gamma-PVs) in pre-malignant cutaneous lesions of immunocompetent individuals using a new broad-spectrum primer system, and identification of HPV210, a novel gamma-PV type. Virology 2018;525:182-91. doi:10.1016/j.virol.2018.09.006.

30. Lin C, Franceschi S, Clifford GM. Human papillomavirus types from infection to cancer in the anus, according to sex and HIV status: a systematic review and meta-analysis. Lancet Infect Dis 2018;18(2):198-206. doi:10.1016/s1473-3099(17) 30653-9. 